Supporting Information

\title{
Nanoparticle Shape Determines Dynamics of Targeting Nanoconstructs on Cell Membranes
}

\author{
Priscilla Choo, ${ }^{\dagger, \S}$, Tingting Liu, ${ }^{\dagger, \S}$, and Teri W. Odom $*, \dagger, \dagger$ \\ ${ }^{\dagger}$ Department of Chemistry and Department of Materials Science and Engineering, \\ Northwestern University, Evanston, Illinois 60208
}

§These authors contributed equally to this work

*Corresponding Author; Email: todom@ northwestern.edu

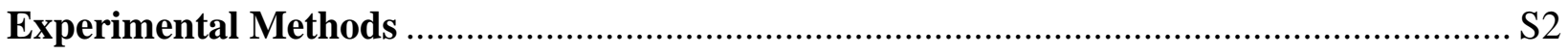

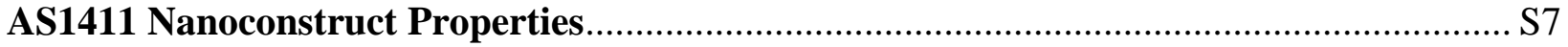

Differentiation of 50-nm Spheres from Cellular Organelles ......................................... S8

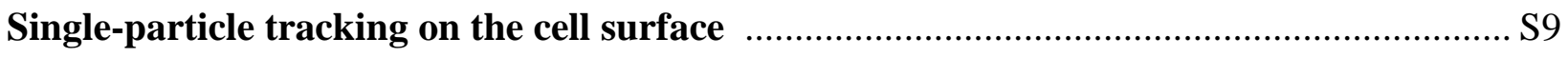

Rotational Motions of AS1411-AuNS on NCL+ and NCL- Cells ................................ S11

Effect of Nucleolin Inhibition on Control AS1411-AuNS Dynamics ..............................S12

The Influence of Endostatin on MCF-7 cell Health ...................................................... S13

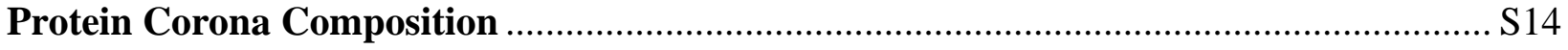

Determination of Single-particle Translational Modes .............................................. S16 


\section{Experimental Methods}

\section{Gold Nanoparticle Synthesis}

All chemicals were purchased from Sigma Aldrich unless otherwise noted. The nanoconstructs were prepared and characterized with previously published protocols. ${ }^{1}$ Gold nanostars (AuNS) were synthesized in lab; 50-nm gold nanospheres (50NPs) were purchased from Ted Pella.

AuNS were synthesized by reducing $\mathrm{HAuCl}_{4}$ with $\mathrm{HEPES}$ buffer (adjusted to $\mathrm{pH}=7.4 \pm 0.02$ with $\mathrm{NaOH}$ pellets from $1 \mathrm{M}$ HEPES buffer) in multiple 20-mL batches. $40-\mathrm{mM} \mathrm{HAuCl}{ }_{4}$ was added to HEPES buffer with the final concentration of 100-mM HEPES and 0.2-mM $\mathrm{HAuCl}_{4}$, and the solution was immediately vortexed for $1 \mathrm{~min}$. The sample was left at room temperature for overnight to allow particle growth and stabilization.

\section{$\underline{\text { AS1411-Nanoconstructs Preparation }}$}

All DNA aptamers were purchased from Integrated DNA technologies (IDT). AS1411 aptamers (5' ThioMC6-D-TT GGT GGT GGT GGT TGT GGT GGT GGT GG 3') were conjugated to AuNS with $20-\mathrm{mM} \mathrm{pH}=3$ citrate buffer at 1600:1 aptamer:AuNS ratio. Bare synthesized AuNS were washed and concentrated 5 times, then AS1411 was added, followed by 5-min incubation. 100-mM citrate buffer stock with added to achieve final concentration of 20 $\mathrm{mM}$. The sample was vortexed to mix and was left on shaker for 16 hours to allow for DNA adsorption and conjugation.

Cy5-AS1411 aptamers (5' ThioMC6-D-isp18-iCy5-TT GGT GGT GGT GGT TGT GGT GGT GGT GG 3') were conjugated onto 50NPs for fluorescence tracking at 1200:1 aptamer:50NP ratio. Aptamers were directly added to bare 50NPs solution. Sodium PBS was first added to reach minimum $\mathrm{Na}$ concentration of $10 \mathrm{mM}$ to prevent aggregation, and the solution was vortexed. The salt-aging solution (4.717 M NaCl, $0.79 \mathrm{mM}$ monosodium phosphate, $9.3 \mathrm{mM}$ sodium phosphate 
dibasic heptahydrate, and $0.01 \%$ TWEEN 20) was added in a step-wise manner twice, until the final sodium ion concentration reached $250 \mathrm{mM}$.

Non-targeting control AS1411 (cAS1411) aptamers (5' ThioMC6-D-TT CCT CCT CCT CCT TCT CCT CCT CCT CC-3SpC3 3') were conjugated to AuNS with 20-mM pH = 4.5 citrate buffer at 16)):1 aptamer:AuNS ratio to achieve similar ligand loading as AS1411. Similar to AS1411 conjugation, the bare particles were washed and concentrated 5 times. cAS1411 solution was then added to concentrated AuNS followed by 5 min incubation; $100-\mathrm{mM} \mathrm{pH}=4.5$ citrate buffer was added to final concentration of $20 \mathrm{mM}$. The sample was left on the shaker at room temperature for 16 hours after vortexing for mixing.

\section{Nanoconstruct Characterization}

The loading for AS1411-AuNS was quantified with Quan-iT ${ }^{\mathrm{TM}}$ OliGreen $^{\mathrm{TM}}$ ssDNA assay (Thermo Fisher Scientific, Waltham, Massachusetts, USA); the loading for AS1411-50NPs was quantified with the luminescence of Cy5. In both cases, the Au core was dissolved with potassium cyanide to avoid influencing the fluorescence signal. The measurement was done with Synergy Neo2 Multi-Mode Microplate Reader (BioTek, Winooski, Vermont, USA).

Hydrodynamic diameters of the nanoconstructs were quantified by dynamic light scattering (DLS); surface charges were compared through $\zeta$-potential. Both were measured with ZetaPALS zeta potential and particle size analyzer (Brookhaven Instruments, Holtsville, New York, USA).

\section{$\underline{\text { Cell Culture }}$}

The adenocarcinoma MCF-7 cell line (ATCC, USA) was maintained in complete growth medium: MEM medium (Corning, Glendale, Arizona, USA) supplemented with $10 \%$ fetal bovine serum (FBS) (Thermal Fisher Scientific, Inc., Waltham, MA, USA) and $0.01 \mathrm{mg} / \mathrm{mL}$ human 
recombinant insulin. The cells were cultured at $37^{\circ} \mathrm{C}$ with $5 \% \mathrm{CO}_{2}$ and plated in $\mathrm{T} 75$ flasks (Corning, Glendale, Arizona, USA).

\section{Cell Viability with Endostatin}

MCF-7 cells were plated into 96-well plate (Corning, Glendale, Arizona, USA) at the density of 20,000 cells/well in $100 \mu \mathrm{L}$ of complete growth medium. After 24 -h incubation at $37^{\circ} \mathrm{C}$ with $5 \% \mathrm{CO}_{2}$, the medium was replaced with endostatin solutions. The endostatin powder was firstly weighed and dissolved with sterile Dulbecco's phosphate-buffered saline (DPBS, Thermal Fisher Scientific, Inc., Waltham, MA, USA) and diluted with complete growth medium and DPBS to different concentrations. The DPBS volume was kept at $10 \%$ of final volume for all concentrations. The MCF-7 cells were incubated with $100-\mu \mathrm{L}$ endostatin solutions per well for 30 min before cell viability assay.

The cell viability for the endostatin-treated cells were quantified with MTS reagent (Promega, Madison, Wisconsin, USA). Endostatin solutions were replaced with 100- $\mu$ L of complete growth medium after 30-min incubation. $20 \mu \mathrm{L}$ of MTS reagent was added in each well before leaving the plate at $37^{\circ} \mathrm{C}$ with $5 \% \mathrm{CO}_{2}$. A negative control was prepared as $20 \mu \mathrm{L}$ MTS reagent was added to $100 \mu \mathrm{L}$ of complete growth medium without MCF-7 cells. After 2-h incubation, $100 \mu \mathrm{L}$ of the mixed sample was transferred to a new plate for each well, and the absorbance was recorded at 490 nm with Synergy Neo2 Multi-Mode Microplate Reader (BioTek, Winooski, Vermont, USA).

\section{Protein Corona Composition Characterization}

The two nanoconstructs, AS1411-AuNS and AS1411-50NPs, were incubated with imaging medium (MEM with 10\% FBS, $0.01 \mathrm{~g} / \mathrm{mL}$ Insulin, and $15 \mathrm{mM}$ HEPES) respectively for $30 \mathrm{~min}$, during which time a protein corona formed. To analyze the composition of the protein corona, the nanoconstructs were separated and washed via centrifugation to remove excess of proteins. The 
denaturing SDS-PAGE was performed using a Xcell Surelock Mini-Cell electrophoresis system (Invitrogen, Carlsbad, CA, USA) with NuPAGE 4-12\% Bis-Tris protein gels (Invitrogen, Carlsbad, CA, USA) according to manufacturer instructions to collect the adsorbed proteins for all. Three replicates for each nanoconstruct composition were prepared to avoid the randomly adsorbed proteins. The collected proteins were quantified with Northwestern Proteomics center. Proteins with less than $10 \%$ of whole sequence detected were not considered to be part of the protein corona profiles.

\section{$\underline{\text { Live-Cell Imaging }}$}

For live-cell imaging, the cells were plated on glass coverslips at the density of 20,000 cells $/ \mathrm{cm}^{2}$ in a customized 2-cm² PDMS cell and allowed to grow for $48 \mathrm{~h}$ at $37^{\circ} \mathrm{C}$ with $5 \% \mathrm{CO}_{2}$. The prepared cell samples were directly used for imaging as nucleolin-overexpressed (NCL+) cells. For the nucleolin-inhibited $\left(\mathrm{NCL}^{-}\right)$case, the MCF-7 cells were incubated with $25 \mu \mathrm{g} / \mathrm{mL}$ endostatin for $30 \mathrm{~min}$ at $37^{\circ} \mathrm{C}$ with $5 \% \mathrm{CO}_{2}$ to bind with the NCL receptor on the cell membranes to prevent the binding with AS1411-nanoconstructs.

As demonstrated in the SI reference 2, cells can be imaged with differential interference contrast (DIC) microscopy with similar S/N at different wavelengths. For AS1411-AuNS and cAS1411-AuNS, DIC images were collected at $\lambda=700 \pm 5 \mathrm{~nm}$, which is close to the localized surface plasmon resonance of the AuNS and within the high-performance wavelength range for the optical components (polarizers and sCMOS camera). For AS1411-50NPs, cells were imaged in the DIC channel at $\lambda=543 \pm 11 \mathrm{~nm}$, where a wide bandwidth filter was used to compensate low lamp light power and detector sensitivity. The particles were tracked through the epifluorescence signal from Cy5 dyes, which were excited with $645 \mathrm{~nm}$, and emission was collected at $689 \mathrm{~nm}$. 
To maintain cell health during the imaging session, 1 M HEPES buffer ( $\mathrm{pH}=7.4$ ) (Atlanta Biologicals, USA) were added to complete growth medium to achieve a final concentration of 15 mM to keep the pH constant. Nanoconstructs, AS1411-AuNS, AS1411-50NPs, or cAS1411-AuNS, were suspended with imaging medium at final concentration of $0.6 \mathrm{nM}$ and incubated for $30 \mathrm{~min}$ before they were introduced into the imaging chamber through perfusion.

All streams were acquired with 100-ms exposure times; each stream consisted of 300 frames. For statistics, only one stream was taken for one particle for all imaging conditions: AS1411-AuNS, AS1411-50NPs, and cAS1411-AuNS with NCL+ and NCL- MCF-7 cells respectively.

\section{Image Processing}

Customized MATLAB programs were written to analyze DIC and/or epifluorescence signals produced by the single nanoconstructs on MCF-7 cells. For AS1411-AuNS and cAS1411-AuNS, the tracking was achieved with the same method in SI reference 2. The nanoconstructs (AS1411AuNS and cAS1411-AuNS) were automatically identified and tracked based on the DIC images with the custom program. Manual clicking was done to minimize the error with the MATLAB program for certain particles when the nanoconstruct underwent long translational movements or was in a complex environment where the cellular background also experienced fast motion. The translational motions of both nanoconstructs were processed with MSD analysis by the determined NP coordinates from particle tracking.

Particle intensity (I) was calculated as the average intensity of a circular AuNS pattern (12 pixels in diameter) around the determined particle location. Background intensity $\left(I_{b k g}\right)$ was defined as the average intensity of a small square pattern ( 31 pixels $\times 31$ pixels $)$ around the tracked particle with AuNS pixels excluded. The DIC contrast of the particles was calculated as $(I-$ $\left.I_{b k g}\right) / I_{b k g}$, which we used to quantify the rotational motions of AuNS nanoconstructs. 


\section{AS1411 Nanoconstruct Properties}

To investigate the impact of nanoconstruct core shape on nanoparticle (NP)-cell interactions, we carefully designed the two nanoconstructs to have the same ligand density. The 50NPs were chosen because they have surface areas similar to $40 \mathrm{~nm}$ AuNS. Through fine control of surface ligand functionalization method, we synthesized AS1411-AuNS and AS1411-50NPs with similar AS1411 densities.

Table S1 summarizes the surface area, ligand loading, ligand density, hydrodynamic diameter, and surface charge for both AS1411-AuNS and AS1411-50NPs. The two nanoconstructs showed similar ligand density and surface charge, which enables us to determine the impact of particle shape on targeted NP-cell interactions.

Table S1. Characterization of AS1411-AuNS and AS1411-50NPs

\begin{tabular}{c|c|c|c|c|c}
\hline Nanoconstructs & SA $\left(\mathrm{nm}^{2}\right)$ & DNA/NC & Density $\left(/ \mathrm{nm}^{2}\right)$ & $\begin{array}{c}\text { Hydrodynamic } \\
\text { Diam. }(\mathrm{nm})\end{array}$ & $\begin{array}{c}\zeta \text {-potential } \\
(\mathrm{mV})\end{array}$ \\
\hline AS1411-AuNS & 7255 & $340 \pm 20$ & 0.0469 & $51.18 \pm 0.28$ & $-37.69 \pm 1.95$ \\
\hline AS1411-50NPs & 7239 & $350 \pm 20$ & 0.0483 & $59.35 \pm 0.36$ & $-37.37 \pm 1.35$ \\
\hline
\end{tabular}




\section{Differentiation of 50-nm Spheres from Cellular Organelles}

Because of their isotropic shape, DIC image patterns of 50NPs are not orientation dependent. Figure S1 shows the importance of fluorescence labeling for the tracking of 50NPs. The DIC image and epifluorescence image were merged during data processing, so the DIC image patterns overlapped with the fluorescence signal were the identified AS1411-50NPs. The red arrows point to AS1411-50NPs in the DIC images, and the white arrows point to cellular features with similar DIC image patterns. The patterns indicate by the two different colored arrows were visually similar, which suggests the difficulty to directly locate AS1411-50NPs in DIC channels.

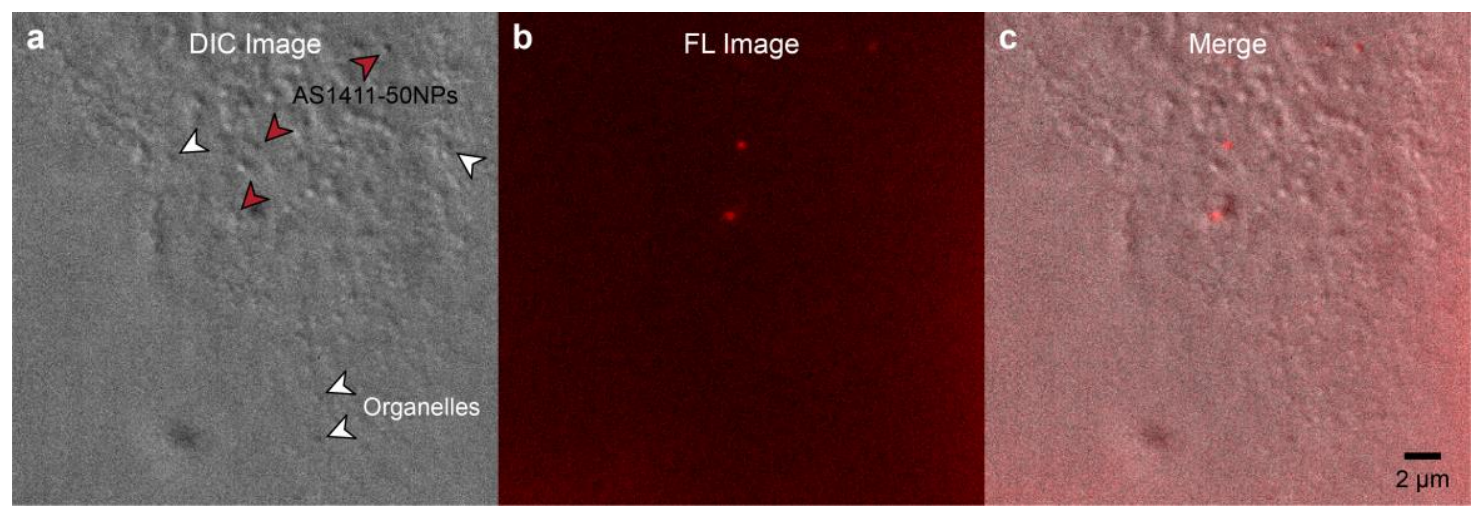

Figure S1. The similarity between 50NPs and cellular features in DIC images. (a) DIC image, (b) epifluorescence image, and (c) overlaid DIC-epifluorescence image for AS1411-50NPs on NCL+ MCF-7 membranes. 


\section{Single-particle tracking on the cell surface}

Z-sectioning of MCF-7 cells (20-30 $\mu$ m thick $)^{3}$ ensured that only AuNS on the cell membrane were tracked. Figure $\mathbf{S 2}$ shows different planes of an island of MCF-7 cells, where the structure of the bottom, middle, and top planes of the cells is clearly different. Imaging planes were carefully selected after z-sectioning of cells. All streams in this work were acquired at the top plane where no features of nucleus or large number of cellular vesicles were visible. Furthermore, all tracked particles stayed in the same focal plane for the whole 30-s stream.

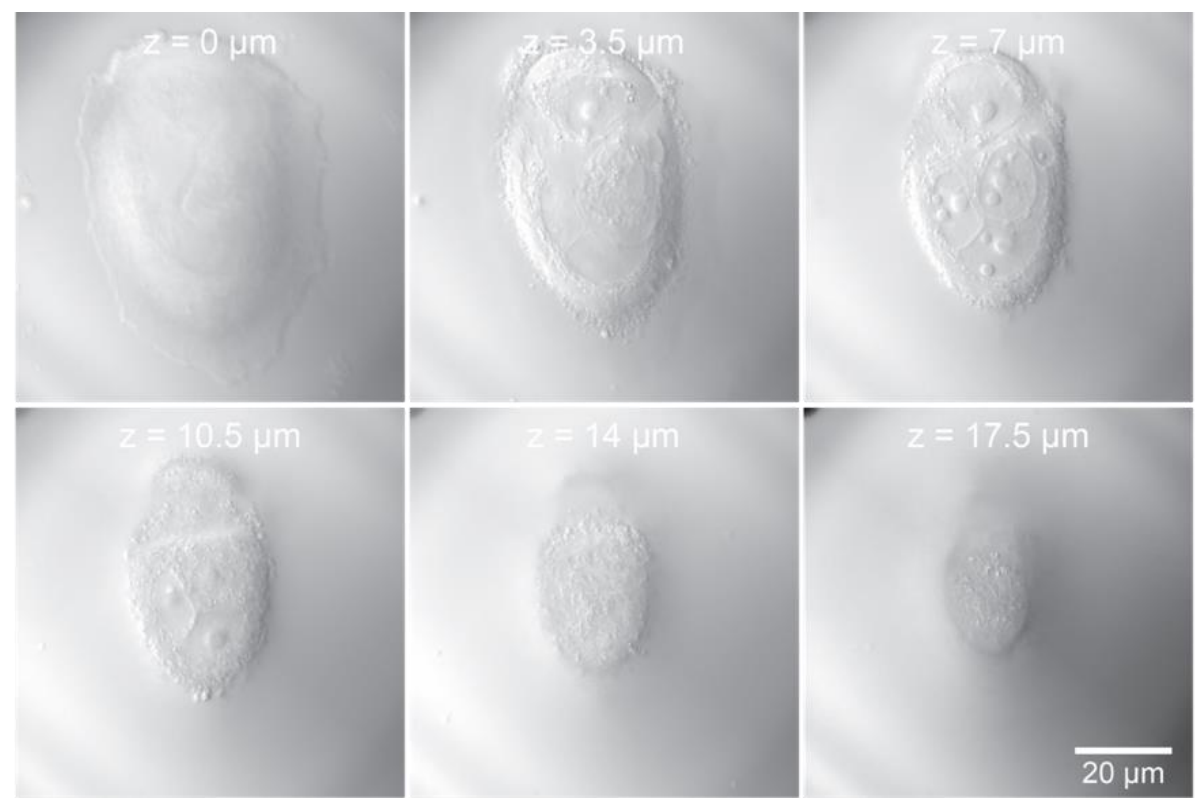

Figure S2. Z-sectioning of MCF-7 cells with DIC imaging. Optical sectioning of MCF-7 cells with DIC imaging show that the axial resolution of DIC can locate the cell membrane for singleparticle tracking on the surface.

Figure S3 shows DIC images of a representative AS1411-AuNS going through intracelluar motion in MCF-7 cells, where the particle does not remain in the same image plane after $7 \mathrm{~s}$. Hence, nanoconstructs whose motion can be followed within the same plane for longer than $30 \mathrm{~s}$ are considered staying on the cell membrane before they are endocytosed. 


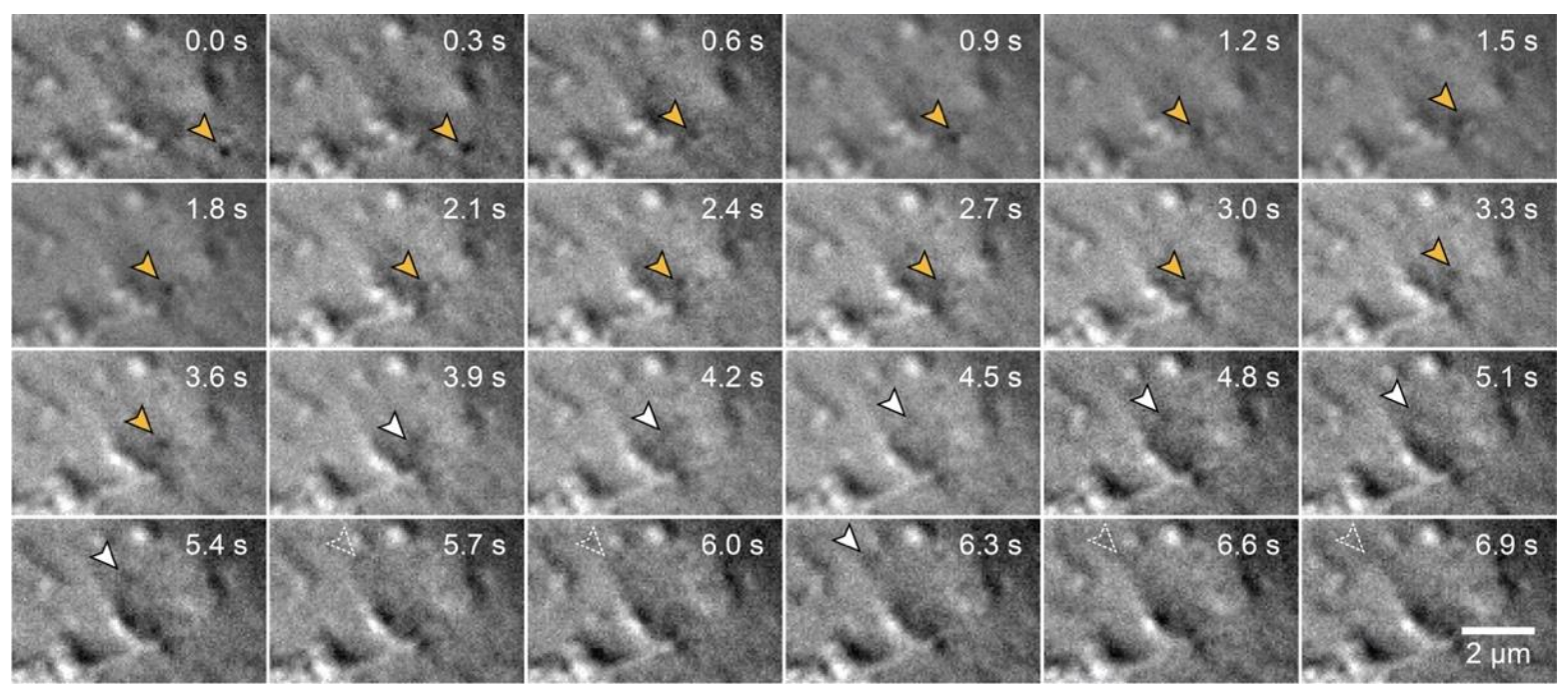

Figure S3. Change of $\mathbf{z}$ height of intracellular particles. DIC images of an internalized particle going through intracellular transport shows fluctuation in $\mathrm{z}$, which can no longer be tracked after 7 seconds. Yellow arrows: particle moving in-plane. White arrows: particles changed in $\mathrm{z}$ height showing blurred DIC patterns. Dashed arrows: particles not visible within the ROI. 


\section{Rotational Motion of AS1411-AuNS on NCL+ and NCL- MCF-7 Cells}

Figure S4 plots the DIC images within the first $5 \mathrm{~s}$ of the 30 -s stream for two representative AS1411-AuNS on NCL+ and NCL- cells. On NCL+ MCF-7 cells, the DIC image pattern of AS1411-AuNS barely changed over the $5 \mathrm{~s}$ (Figure S2a). The little change in DIC image patterns suggests that AS1141-AuNS did not rotate much over this time period. In contrast, the DIC pattern fluctuated rapidly between bright and dark when the particle was on NCL- MCF-7 membranes, which indicates large angular changes during AS1411-AuNS rotation. Since single-particle rotation reflects ligand/receptor interactions and receptor clustering, ${ }^{4}$ we attributed the increased angular change during AS1411-AuNS rotation on NCL- cells to the unavailability of NCL for binding. Therefore, we confirmed that AS1411-NCL interactions determine the single-particle dynamics of AS1411-AuNS on NCL+ cells.

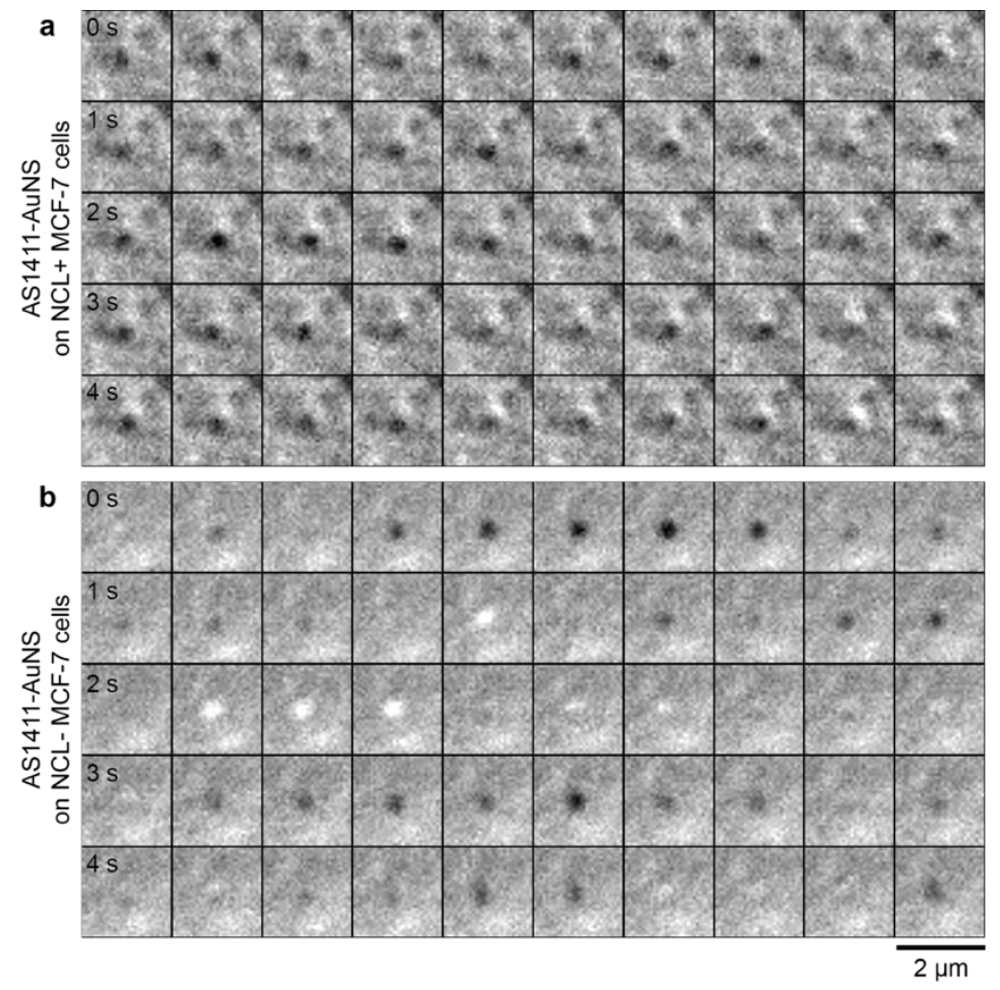

Figure S4. DIC images for AS1411-AuNS on NCL+ and NCL- MCF-7 cells for the first $5 \mathrm{~s}$ of the streams. AS1411-AuNS on (a) NCL+ and (b) NCL- MCF-7. 


\section{Effect of Nucleolin Inhibition on Control AS1411-AuNS Dynamics}

To investigate whether the treatment of endostatin changed MCF-7 cell behavior, we compared the rotational motions of cAS1411-AuNS on NCL+ and NCL- MCF-7 cells (Figure S5). Two representative cAS1411-AuNS showed fast variation from bright to dark DIC patterns throughout the whole stream. The contrast plots in Figure S3b showed that contrast rapidly fluctuated around 0 for both particles, which reflects changes in DIC image patterns. We quantitatively compared the rotational dynamics through maximum contrast duration (Figure S5c), and no significant difference was observed for $\mathrm{NCL}+$ and $\mathrm{NCL}-$ cases $(p=0.894)$. The constant rotational dynamics of cAS1411-AuNS suggests that the cell behavior did not change after NCL inhibition.
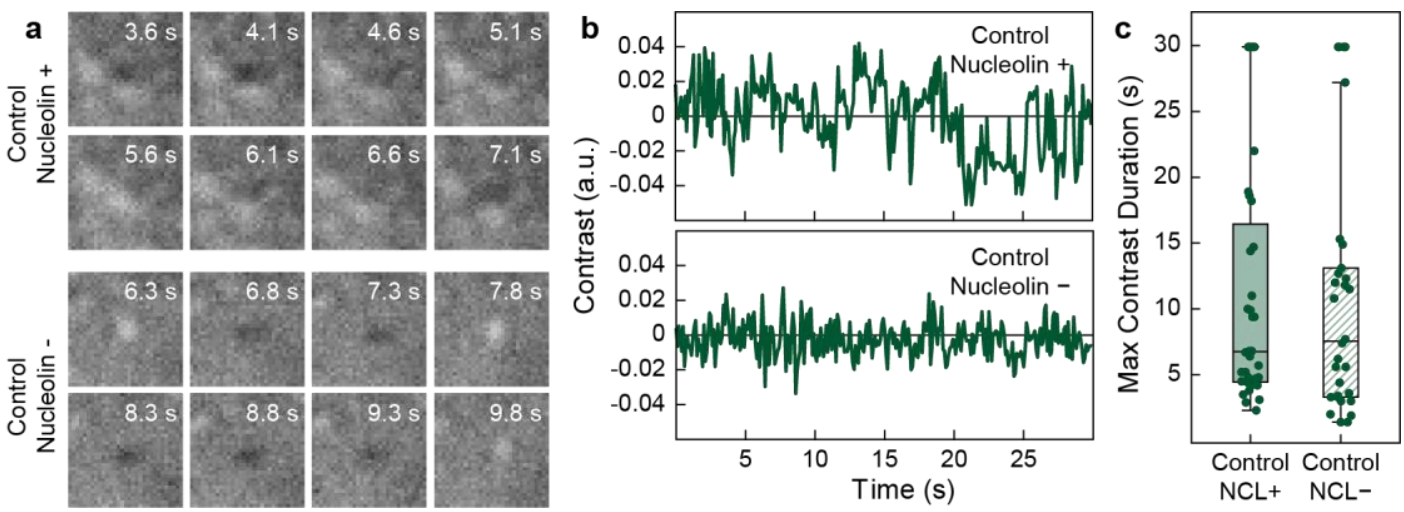

Figure S5. Rotational dynamics of cAS1411-AuNS. (a) DIC images of cAS1411-AuNS on $\mathrm{NCL}+$ and NCL - cell membrane (b) Plot of contrast over time for particles in (a). (c) Comparison of maximum duration for cAS1411-AuNS on NCL+ and NCL- MCF-7 cells. $(p=0.894)$ 


\section{The Influence of Endostatin on MCF-7 cell health}

Endostatin is used as an inhibitor to prevent the binding between AS1411 and NCL receptors. ${ }^{5}$ We first determined the proper concentration for NCL inhibition in our experiment. Figure S6 shows the viability of MCF-7 cells treated with different concentrations of endostatin. The viability of MCF-7 cells remained higher than $95 \%$ at the highest concentration. Therefore, $25 \mu \mathrm{g} / \mathrm{mL}$ endostatin was incubated with MCF-7 cells for 30 min prior to imaging to prepare NCL- MCF-7 cells.

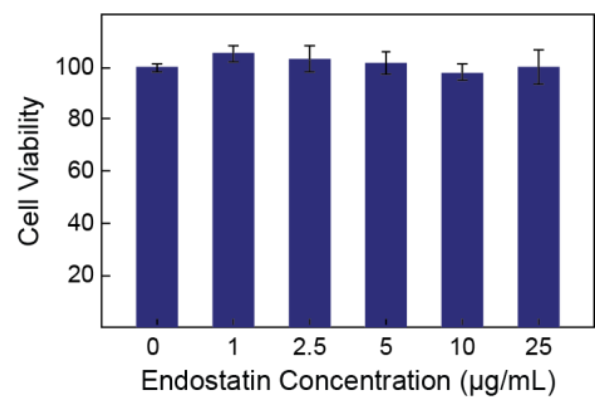

Figure S6. Viability of MCF-7 cells after endostatin treatment. Cell health was maintained at all endostatin concentrations. 


\section{Protein Corona Composition}

We investigated whether the nanoparticle shape would affect the composition of the protein corona when the surface ligands were kept the same. Figure S7 shows the Venn diagram for the protein corona composition for AS1411-AuNS and AS1411-50NPs, where the protein types within the corona were almost identical for both nanoconstructs. 62 different types of proteins were shared by AS1411-AuNS and AS1411-50NPs. Six unique proteins were reported for AS1411-AuNS; ten unique proteins were reported for AS1411-50NPs. None of the unique protein compositions for both nanoconstructs has been reported to impact cell-targeting functions in previous literature.

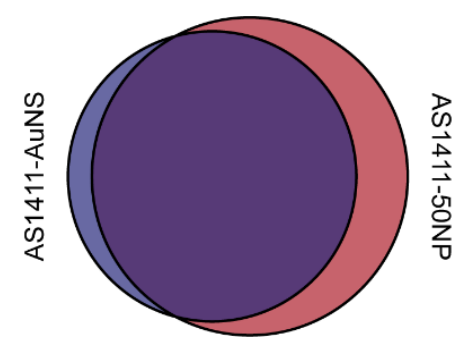

Figure S7. Protein corona composition comparison between AS1411-AuNS and AS141150NP. The protein corona composition is similar for both nanoconstructs.

Beside protein types, we also compared the quantity of each protein component in the corona for both nanoconstructs. Table S2 summarizes the top 20 proteins within the protein corona for AS1411-AuNS and AS1411-50NPs ordered by relative protein abundance. The highlighted boxes indicate the shared proteins. Serum albumin was the most abundant protein for both and was the only protein that accounted for more that $10 \%$ relative abundance. Table S2 shows that not only the protein type was consistent between the two constructs, but also quantities of different proteins were similar. With almost identical protein corona compositions, we hypothesized that NP-cell interactions would be similar if the protein corona was the dominant factor to determine particlecell interactions. Therefore, the difference observed in single particle dynamics can be attributed to the particle shape. 
Table S2. Most abundant (top 20) proteins identified in the in vitro protein corona of AS1411-AuNS, AS1411-50NPs, and cAS1411-AuNS. Relative protein abundance (RPA) values represent the average from three replicates.

\begin{tabular}{|c|c|c|c|}
\hline \multicolumn{2}{|l|}{ AS1411-AuNS } & \multicolumn{2}{|l|}{ AS1411-50NPs } \\
\hline Protein Name & RPA & Protein Name & RPA \\
\hline Serum albumin & $22.51 \%$ & Serum albumin & $17.71 \%$ \\
\hline $\begin{array}{c}\text { Alpha-1- } \\
\text { antiproteinase }\end{array}$ & $7.97 \%$ & $\begin{array}{c}\text { Alpha-1- } \\
\text { antiproteinase }\end{array}$ & $8.82 \%$ \\
\hline Serotransferrin & $7.30 \%$ & $\begin{array}{l}\text { Alpha-2-HS- } \\
\text { glycoprotein }\end{array}$ & $6.00 \%$ \\
\hline $\begin{array}{l}\text { Alpha-2-HS- } \\
\text { glycoprotein }\end{array}$ & $7.15 \%$ & Serotransferrin & $5.89 \%$ \\
\hline Serotransferrin & $6.93 \%$ & Serotransferrin & $5.72 \%$ \\
\hline Complement C3 & $2.91 \%$ & $\begin{array}{c}\text { Alpha-2- } \\
\text { macroglobulin }\end{array}$ & $3.55 \%$ \\
\hline Alpha-fetoprotein & $2.56 \%$ & Complement C3 & $3.40 \%$ \\
\hline $\begin{array}{c}\text { Alpha-2- } \\
\text { macroglobulin }\end{array}$ & $2.31 \%$ & Antithrombin-III & $2.76 \%$ \\
\hline ITIH2 protein & $1.84 \%$ & Alpha-fetoprotein & $2.62 \%$ \\
\hline $\begin{array}{c}\text { Vitamin D-binding } \\
\text { protein }\end{array}$ & $1.49 \%$ & Complement factor $\mathbf{H}$ & $2.25 \%$ \\
\hline Serpin A3-1 & $1.41 \%$ & Serpin A3-1 & $1.82 \%$ \\
\hline Antithrombin-III & $1.33 \%$ & $\begin{array}{c}\text { Uncharacterized } \\
\text { protein }\end{array}$ & $1.64 \%$ \\
\hline $\begin{array}{l}\text { Inter-alpha-trypsin } \\
\text { inhibitor heavy chain } \\
\text { H4 }\end{array}$ & $1.33 \%$ & ITIH2 protein & $1.60 \%$ \\
\hline Complement factor $\mathbf{H}$ & $1.19 \%$ & $\begin{array}{c}\text { Inter-alpha-trypsin } \\
\text { inhibitor heavy chain } \\
\text { H4 }\end{array}$ & $1.22 \%$ \\
\hline Plasminogen & $1.12 \%$ & $\begin{array}{c}\text { Vitamin D-binding } \\
\text { protein }\end{array}$ & $1.20 \%$ \\
\hline $\begin{array}{c}\text { Inter-alpha-trypsin } \\
\text { inhibitor heavy chain } \\
\text { H3 }\end{array}$ & $1.11 \%$ & Plasminogen & $1.07 \%$ \\
\hline Fetuin-B & $1.06 \%$ & Plasminogen & $0.98 \%$ \\
\hline Complement factor B & $1.05 \%$ & Fetuin-B & $0.96 \%$ \\
\hline Actin, cytoplasmic 1 & $0.94 \%$ & $\begin{array}{c}\text { Uncharacterized } \\
\text { protein }\end{array}$ & $0.96 \%$ \\
\hline $\begin{array}{l}\text { Uncharacterized } \\
\text { protein }\end{array}$ & $0.79 \%$ & $\begin{array}{c}\text { Inter-alpha-trypsin } \\
\text { inhibitor heavy chain } \\
\text { H3 }\end{array}$ & $0.92 \%$ \\
\hline
\end{tabular}




\section{Determination of Single-Particle Translational Modes}

The translational motion of single nanoconstructs was processed by mean square displacement (MSD) analysis calculated based on particle coordinates in each frame. We characterized the motions of single nanoconstructs into four different modes $^{6}$ : stationary(ST), simple diffusion (SD), directed diffusion (DD), and restricted diffusion (RD). The second to fourth MSD data points, corresponding to time difference $(\Delta t=0.2 \mathrm{~s}, 0.3 \mathrm{~s}$, and $0.4 \mathrm{~s})$, were fit to a linear curve. Particles with a fitted slope less than $1.84 \times 10^{-3} \mu \mathrm{m}^{2} / \mathrm{s}$ were categorized as stationary particles.
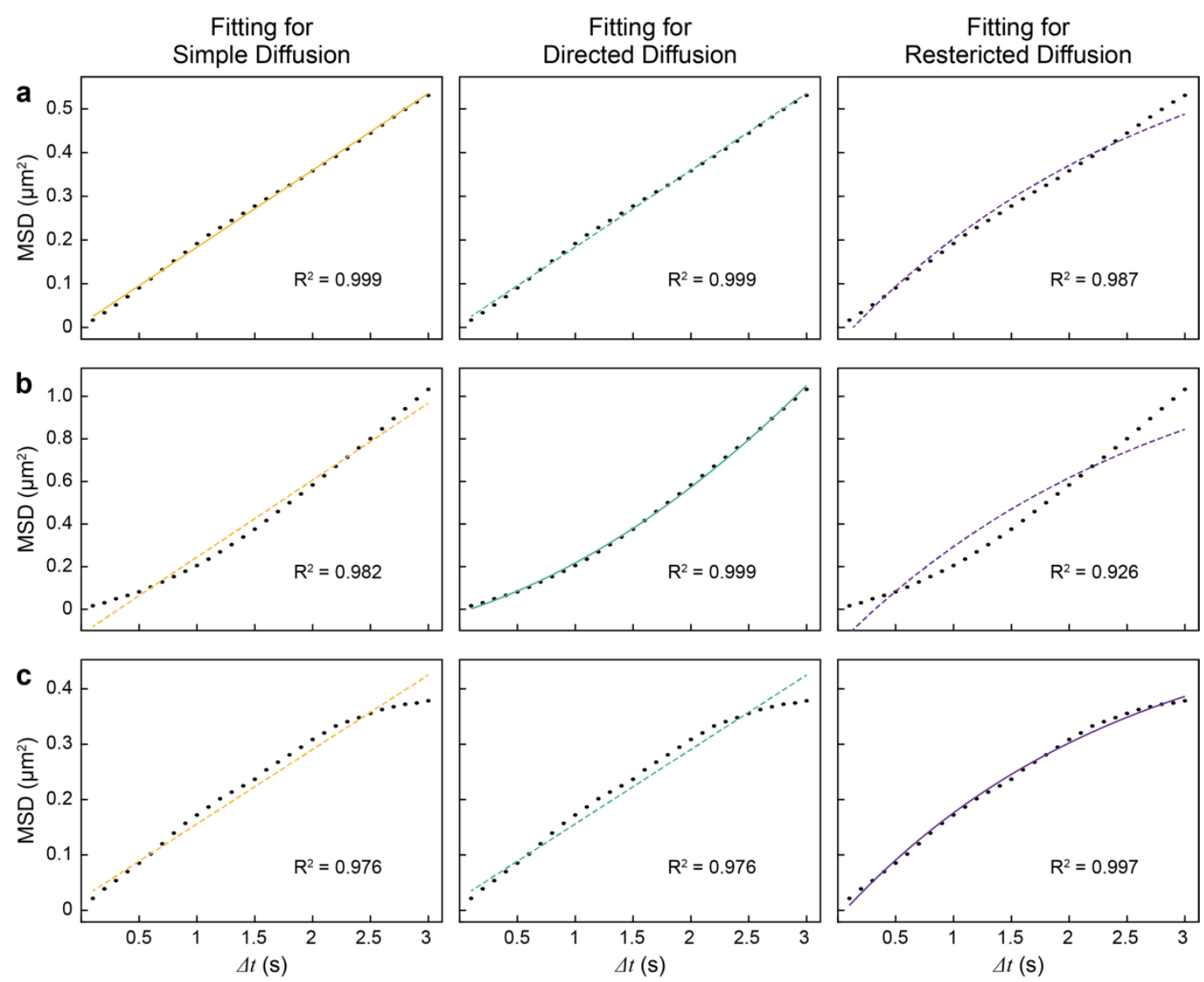

Figure S8. Determination of translational mode. Fitting curve for three particles categorized as (a) simple diffusion, (b) directed diffusion, and (c) restricted diffusion.

For non-stationary particles, the first 30 MSD data points, where the time difference is no longer than $3 \mathrm{~s}$, were fit to three equations for simple diffusion (Equation S2), directed diffusion (Equation S3), and restricted diffusion (Equation S4) respectively shown as following: 
$\operatorname{MSD}(\Delta t)=4 D \Delta t$

(Equation S2)

$\operatorname{MSD}(\Delta t)=4 D \Delta t+v^{2}(\Delta t)^{2}$

(Equation S3)

$\operatorname{MSD}(\Delta t)=\frac{L^{2}}{3}\left\{1-\exp \left(\frac{-12 D \Delta t}{L^{2}}\right)\right\}$

(Equation S4)

$\mathrm{R}^{2}$ for the three fittings were calculated and compared for the same particle (Figure S8). The translational mode was assigned to the fitting with largest $R^{2}$ value, since largest $R^{2}$ value indicated the equation best described the translational motions of the particle within the 30-s stream. For Figure S6a, the particle has a velocity $(v)$ of $0.00223 \mu \mathrm{m} / \mathrm{s}$ with Equation S3, two orders of magnitude smaller than $0.130 \pm 0.056 \mu \mathrm{m} / \mathrm{s}$, the average velocity for DD AS1411-AuNS. Therefore, the second term in the Equation S3 does not contribute to the overall fit, resulting in similar $\mathrm{R}^{2}$ values when fit to $\mathrm{SD}$. We assigned $\mathrm{SD}$ to this particle because the $\mathrm{R}^{2}$ value (SD: 0.998664721, DD: 0.998663532) is highest when fit to SD. 


\section{Supporting Information References}

1. Chandra, K.; Culver, K. S. B.; Werner, S. E.; Lee, R. C.; Odom, T. W., Manipulating the Anisotropic Structure of Gold Nanostars using Good's Buffers. Chem Mater 2016, 28 (18), 67636769.

2. Bhowmik, D.; Culver, K. S. B.; Liu, T.; Odom, T. W., Resolving Single-Nanoconstruct Dynamics during Targeting and Nontargeting Live-Cell Membrane Interactions. ACS Nano 2019, 13 (12), 13637-13644.

3. Morita, M.; Tanaka, H.; Kumamoto, Y.; Nakamura, A.; Harada, Y.; Ogata, T.; Sakaguchi, K.; Taguchi, T.; Takamatsu, T., Fluorescence-based discrimination of breast cancer cells by direct exposure to 5-aminolevulinic acid. Cancer Med 2019, 8 (12), 5524-5533.

4. Xiao, L.; Wei, L.; Liu, C.; He, Y.; Yeung, E. S., Unsynchronized translational and rotational diffusion of nanocargo on a living cell membrane. Angew Chem Int Ed Engl 2012, 51 (17), 4181-4.

5. Wu, J.; Song, C.; Jiang, C.; Shen, X.; Qiao, Q.; Hu, Y., Nucleolin Targeting AS1411 Modified Protein Nanoparticle for Antitumor Drugs Delivery. Molecular Pharmaceutics 2013, 10 (10), 3555-3563.

6. Kusumi, A.; Sako, Y.; Yamamoto, M., Confined lateral diffusion of membrane receptors as studied by single particle tracking (nanovid microscopy). Effects of calcium-induced differentiation in cultured epithelial cells. Biophysical Journal 1993, 65 (5), 2021-2040. 